\title{
Plasma Nucleosomes in Primary Breast Cancer
}

\author{
Michal Mego 1,2,*®D, Katarina Kalavska ${ }^{2}$, Marian Karaba ${ }^{3}$, Gabriel Minarik ${ }^{4}$, Juraj Benca ${ }^{3,5}$, \\ Tatiana Sedlackova ${ }^{4}$, Paulina Gronesova ${ }^{6}$, Dana Cholujova ${ }^{6}$, Daniel Pindak ${ }^{3,7}$, Jozef Mardiak ${ }^{1}$ \\ and Peter Celec ${ }^{4}(\mathbb{D}$
}

1 2nd Department of Oncology, Faculty of Medicine, Comenius University and National Cancer Institute, 83310 Bratislava, Slovakia; jmardiak@gmail.com

2 Translational Research Unit, Faculty of Medicine, Comenius University and National Cancer Institute, 83310 Bratislava, Slovakia; katarina.kalavska@nou.sk

3 Department of Oncosurgery, National Cancer Institute, 83310 Bratislava, Slovakia; marian.karaba@nou.sk (M.K.); juraj.benca@nou.sk (J.B.); daniel.pindak@nou.sk (D.P.)

4 Institute of Molecular Biomedicine, Faculty of Medicine, Comenius University, 81372 Bratislava, Slovakia; gabriel.minarik@gmail.com (G.M.); tatiana.sedlackova@gmail.com (T.S.); petercelec@gmail.com (P.C.)

5 Department of Medicine, St. Elizabeth University, 81102 Bratislava, Slovakia

6 Biomedical Center, Slovak Academy of Sciences, 84505 Bratislava, Slovakia; paulina.gronesova@gmail.com (P.G.); dana.cholujova@savba.sk (D.C.)

7 Department of Oncosurgery, Slovak Medical University, 83101 Bratislava, Slovakia

* Correspondence: misomego@gmail.com or michal.mego@nou.sk; Tel.: +421-2-59378366; Fax: +421-2-54774943

Received: 7 August 2020; Accepted: 8 September 2020; Published: 10 September 2020 updates

Simple Summary: Nucleosomes composed of DNA and histone proteins enter the extracellular space and end eventually in the circulation when cells die. In blood plasma, they could represent a nonspecific marker of cell death, potentially useful for noninvasive monitoring of cancer. The aim of this study was to analyze circulating nucleosomes in relation to patient/tumor characteristics and prognosis in nonmetastatic breast cancer. This study included 92 patients with breast cancer treated with surgery. Plasma nucleosomes were detected in samples taken in the morning on the day of surgery. Circulating nucleosomes were positively associated with the systemic inflammation but not with other patient/tumor characteristics. Patients with lower nucleosomes had lower risk of disease recurrence compared to patients with higher nucleosomes. Our data suggest that plasma nucleosomes in nonmetastatic breast cancer are associated with systemic inflammation and might have a prognostic value. The underlying mechanisms require further studies.

\begin{abstract}
When cells die, nucleosomes composed of DNA and histone proteins enter the extracellular space and end eventually in the circulation. In plasma, they might serve as a nonspecific marker of cell death, potentially useful for noninvasive monitoring of tumor dynamics. The aim of this study was to analyze circulating nucleosomes in relation to patient/tumor characteristics and prognosis in primary breast cancer. This study included 92 patients with breast cancer treated with surgery for whom plasma isolated was available in the biobank. Plasma nucleosomes were detected in samples taken in the morning on the day of surgery using Cell Death Detection ELISA kit with anti-histone and anti-DNA antibodies. Circulating nucleosomes were positively associated with the systemic inflammatory index (SII), but not with other patient/tumor characteristics. Patients with high SII in comparison to low SII had higher circulating nucleosomes (by 59\%, $p=0.02$ ). Nucleosomes correlated with plasma plasminogen activator inhibitor-1, IL-15, IL-16, IL-18, and hepatocyte growth factor. Patients with lower nucleosomes had significantly better disease-free survival ( $H R=0.46$, $p=0.05$ ). In a multivariate analysis, nucleosomes, hormone receptor status, HER2 status, lymph node involvement, and tumor grade were independent predictors of disease-free survival. Our data suggest that plasma nucleosomes in primary breast cancer are associated with systemic inflammation and might have a prognostic value. The underlying mechanisms require further studies.
\end{abstract}


Keywords: primary breast cancer; circulating nucleosomes; circulating tumor cells; plasminogen activator inhibitor-1; cytokines

\section{Introduction}

Breast cancer is the most common diagnosed cancer and the leading cause of cancer death among women in developed countries [1]. Despite advances in cancer prevention, diagnoses, and treatment, still approximately $5 \%$ of patients are diagnosed with metastatic disease, and $20-30 \%$ of initially primary breast cancer develops metastasis subsequently, during the course of the disease.

Extracellular DNA (ecDNA), also called cell-free DNA, is present in blood plasma in various forms [2]. EcDNA in the circulation of cancer patients contains tumor DNA from the primary tumor, metastasis, or circulating tumor cells, as well as healthy host cells mostly of hematopoietic origin [3-5]. Plasma ecDNA is partially free unbound DNA and, so, sensitive to rapid cleavage, but it also can be protected as ecDNA hidden in apoptotic bodies and/or bound to proteins such as histones in the form of nucleosomes [5].

Nucleosomes are composed of DNA wound around histone proteins and represent the basic structural unit of chromatin in the nucleus [6]. After cell death, membranes and nuclei disintegrate and cell-free nucleosomes can get into the circulation. Plasma nucleosomes might serve as a nonspecific biomarker of cell death [7]. This might be of interest in patients not only with autoimmune diseases, but also with sepsis or cancer [8-10]. The prognostic value of the concentration of circulating nucleosomes was shown in several types of cancer including lung, pancreatic, or colorectal cancer [11-15]. For example, in pancreatic cancer, high nucleosome levels during treatment, but not pretherapeutic levels, correlate with time to progression [16]. Similarly, in non-small cell lung cancer, high baseline nucleosome level and/or during chemotherapy was associated with poor response to treatment and these data suggested that circulating nucleosomes are a valuable tool for early prediction of chemotherapy efficacy in cancer patients [17]. However, when it comes to primary breast cancer, data in the published literature are limited.

In this study, we aimed to analyze circulating nucleosomes in relation to patients/tumor characteristics and prognosis in primary breast cancer.

\section{Methods}

\subsection{Study Patients}

This study included 92 primary breast cancer patients (stage I-III) treated with surgery from March to November 2012, for whom plasma isolated in the morning on the day of surgery was available in the biobank. This study represents a substudy of a translational trial that aimed to evaluate prognostic value of circulating tumor cells in primary breast cancer [18]. Study eligibility criteria and study details were described previously [18]. The study was approved by the Institutional Review Board (IRB) of the National Cancer Institute of Slovakia (TRUSK002, 20.6.2011). Each participant provided signed informed consent before study enrollment.

\subsection{Detection of Circulating Tumor Cells (CTCs) in Peripheral Blood}

CTCs were detected in peripheral blood by a quantitative real-time polymerase chain reaction (qRT-PCR)-based assay of peripheral blood as described previously [18-20].

\subsection{Plasma Isolation}

Venous peripheral blood samples were collected in EDTA-treated tubes in the morning on the day of surgery and centrifuged at $1000 \times g$ for $10 \mathrm{~min}$ at room temperature within $2 \mathrm{~h}$ of venipuncture and processed as described previously [21]. 


\subsection{Quantification of Circulating Nucleosomes}

The commercially available Cell Death Detection kit (Roche, Basel, Switzerland) was used for the measurement of nucleosomes. Briefly, $20 \mathrm{~mL}$ of plasma was mixed with biotin-labeled anti-histone and peroxidase-conjugated anti-DNA antibodies. After incubation and washing, the substrate for the peroxidase enzyme was added. Absorbance was measured at $405 \mathrm{~nm}$ in arbitrary units after stopping the reaction. Interassay and intra-assay coefficients of variation were below $10 \%$ and $5 \%$, respectively.

\subsection{Measurement of DD, TF, uPA, and PAI-1 in Plasma}

Plasma tissue factor (TF), d-dimer (DD), urokinase plasminogen activator (uPA), and plasminogen activator inhibitor-1 (PAI-1) were analyzed using enzyme-linked immunosorbent assays (ELISA) as described previously [21].

\subsection{Plasma Cytokines and Angiogenic Factors Analysis}

Plasma samples were analyzed for 51 plasma cytokines and angiogenic factors: TGF- $\beta 1$, TGF- $\beta 2$, TGF- $\beta 3$, IFN- $\alpha 2$, IL-1 $\alpha$, IL-2R $\alpha$, IL-3, IL-12p40, IL-16, IL-18, CTACK, Gro- $\alpha$, HGF, LIF, MCP-3, M-CSF, MIF, MIG, $\beta$-NGF, SCF, SCGF- $\beta$, SDF- $1 \alpha$, TNF- $\beta$, TRAIL, IL-1 $\beta$, Il-1RA, IL-2, IL-4, IL-5, IL-6, IL-7, IL-8, IL-9, IL-10, IL-12, IL-13, IL-15, IL-17, Eotaxin, FGF basic, G-CSF, GM-CSF, IFN- $\gamma$, IP-10, MCP-1, MIP-1 $\alpha$, MIP-1 $\beta$, PDGF bb, RANTES, TNF- $\alpha$, VEGF using predesigned panels as described previously and were available for subset of patients (Bio-Plex Pro TGF- $\beta$ assay, Bio-Plex Pro Human Cytokine 21- and 27-plex immunoassays; Bio-Rad Laboratories, Hercules, CA, USA) [22]. The large panel of cytokines was analyzed as data were available from the previous study [22].

\subsection{Complete Blood Count and Inflammation-Based Scores}

Complete blood count $(\mathrm{CBC})$ and $\mathrm{CBC}$-derived inflammation-based scores were calculated as described previously $[23,24]$. For CBC-derived inflammation-based scores, identical cut-off values as published previously for metastatic breast cancer patients were used [23,24]. Data for calculation of NLR, PLR, MLR, SII were available for 54, 52, 48, and 52 patients, respectively.

\subsection{Statistical Analysis}

The characteristics of patients is summarized using mean (range) for continuous variables and frequency (percentage) for categorical variables. The median follow-up period was calculated as the median observation time among all patients and among those who were still alive at the time of their last follow-up. Disease-free survival (DFS) was calculated from the date of blood sampling to the date of disease recurrence (locoregional or distant), secondary cancer, death, or last follow-up. DFS was estimated using the Kaplan-Meier product limit method and compared between groups by log-rank test. For survival analysis, circulating nucleosomes were dichotomized to "low" or "high" (nucleosome level below vs. above mean, respectively). Univariate analyses with Chi squared or Fisher's exact test were performed to find associations between prognostic factors.

A multivariate Cox proportional hazards model for DFS was used to assess differences in outcome on the basis of the nucleosomes status (above mean vs. below mean), hormone receptor status (positive for either vs. negative for both), HER-2 status (positive or negative), axillary lymph node involvement (N0 vs. $\mathrm{N}+$ ), grade (grade 3 vs. grade 1 and 2). Stepwise regression techniques were used to build multivariate models using a significance level of 0.10 to remain in the model. All $p$ values presented are two-sided, and associations were considered significant if the $\mathrm{p}$ value was less than or equal to 0.05 . Statistical analyses were performed using NCSS 11 Statistical Software (2016, NCSS, LLC., Kaysville, UT, USA, ncss.com/software/ncss). 


\section{Results}

\subsection{Patients' Characteristics}

The study population consisted of 92 primary breast cancer patients with a median age of 60 years (range: 25-83 years). The patient characteristics are shown in Table 1 . There were $79(85.9 \%)$ patients with estrogen receptor-positive (ER) and/or progesterone receptor-positive (PR) tumors, and 16 (17.4\%) patients with HER2/neu-positive tumors.

Table 1. Patients' characteristics.

\begin{tabular}{|c|c|c|}
\hline Variable & $\mathbf{N}$ & $\%$ \\
\hline All Patients & 92 & 100.0 \\
\hline \multicolumn{3}{|l|}{ T-stage } \\
\hline $\mathrm{T} 1$ & 58 & 63.0 \\
\hline$>\mathrm{T} 1$ & 34 & 37.0 \\
\hline \multicolumn{3}{|l|}{ Histology } \\
\hline IDC & 76 & 82.6 \\
\hline other & 16 & 17.4 \\
\hline \multicolumn{3}{|l|}{ Grade } \\
\hline low and intermediate & 49 & 53.3 \\
\hline high grade & 41 & 44.6 \\
\hline unknown & 2 & 2.2 \\
\hline \multicolumn{3}{|l|}{ Lymph nodes } \\
\hline No & 57 & 62.0 \\
\hline $\mathrm{N}+$ & 34 & 37.0 \\
\hline unknown & 1 & 1.1 \\
\hline \multicolumn{3}{|l|}{ LVI } \\
\hline present & 69 & 75.0 \\
\hline absent & 23 & 25.0 \\
\hline \multicolumn{3}{|c|}{ Hormone receptor status (cut-off $\mathbf{1 \%}$ ) } \\
\hline negative for both & 13 & 14.1 \\
\hline positive for either & 79 & 85.9 \\
\hline \multicolumn{3}{|c|}{ Estrogen receptor-positive (cut-off $1 \%$ ) } \\
\hline negative & 16 & 17.4 \\
\hline positive & 76 & 82.6 \\
\hline \multicolumn{3}{|c|}{ Progesterone receptor-positive (cut-off } \\
\hline negative & 25 & 27.2 \\
\hline positive & 67 & 72.8 \\
\hline \multicolumn{3}{|l|}{ HER2 status } \\
\hline positive & 16 & 17.4 \\
\hline negative & 76 & 82.6 \\
\hline \multicolumn{3}{|l|}{ P53 status } \\
\hline negative & 59 & 64.1 \\
\hline positive & 32 & 34.8 \\
\hline unknown & 1 & 1.1 \\
\hline \multicolumn{3}{|l|}{ BCL-2 } \\
\hline negative & 27 & 29.3 \\
\hline positive & 65 & 70.7 \\
\hline \multicolumn{3}{|l|}{ unknown } \\
\hline \multicolumn{3}{|l|}{ Ki67 status (cut-off 14\%) } \\
\hline$<14 \%$ & 48 & 52.2 \\
\hline$>14 \%$ & 44 & 47.8 \\
\hline
\end{tabular}


Table 1. Cont

\begin{tabular}{ccc}
\hline Variable & $\mathbf{N}$ & $\mathbf{\%}$ \\
\hline Molecular subtype & & \\
Luminal A & 43 & 46.7 \\
Luminal B & 36 & 39.1 \\
HER2+ & 1 & 1.1 \\
Triple-negative (TN) & 12 & 13.0 \\
\hline CTC EP & & \\
negative & 75 & 81.5 \\
positive & 17 & 18.5 \\
\hline CTC EMT & & \\
negative & 76 & 17.4 \\
positive & 16 & \\
CTC ANY & & 67.4 \\
negative & 62 & 32.6 \\
positive & 30 & \\
\hline
\end{tabular}

Abbreviations: CTC EP, circulating tumor cells with epithelial phenotype; CTC EMT, circulating tumor cells with epithelial-mesenchymal transition phenotype; CTC ANY, circulating tumor cells irrespective of phenotype; LVI, lymphovascular invasion.

\subsection{Association between Nucleosomes and Patient/Tumor Characteristics}

The characteristics of patients and the associations with circulating nucleosomes are shown in Table 2. The concentration of circulating nucleosomes was not associated with any patient/tumor characteristics except the systemic inflammatory index (SII), where patients with high SII had significantly higher levels of circulating nucleosomes compared to patients with low SII (0.17 vs. $0.27, p=0.02$ ). There was also a trend for higher level of circulating nucleosomes in patients with high neutrophil/lymphocyte ratio $(p=0.07)$. There was no association between molecular subtype and plasma nucleosomes, even if molecular subtypes of breast cancer were further segregated by tumor grade. We also analyzed association of chronic medication/comorbidities (Appendix A, Table A1) and circulating nucleosomes, but we found no association.

Table 2. Association between nucleosomes and patient/tumor characteristics.

\begin{tabular}{|c|c|c|c|c|c|}
\hline Variable & $\mathbf{N}$ & Mean & SEM & Median & $p$-Value \\
\hline All & 92 & 0.18 & 0.02 & 0.13 & NA \\
\hline \multicolumn{6}{|l|}{ T-stage } \\
\hline $\mathrm{T} 1$ & 58 & 0.20 & 0.02 & 0.14 & 0.30 \\
\hline$>\mathrm{T} 1$ & 34 & 0.15 & 0.03 & 0.13 & \\
\hline \multicolumn{6}{|l|}{ Histology } \\
\hline invasive ductal carcinoma & 76 & 0.19 & 0.02 & 0.13 & 0.61 \\
\hline other & 16 & 0.15 & 0.04 & 0.13 & \\
\hline \multicolumn{6}{|l|}{ Grade } \\
\hline low and intermediate & 49 & 0.20 & 0.03 & 0.14 & 0.91 \\
\hline high grade & 41 & 0.16 & 0.03 & 0.13 & \\
\hline unknown & 2 & & & & \\
\hline \multicolumn{6}{|l|}{ Lymph nodes } \\
\hline N0 & 57 & 0.18 & 0.02 & 0.12 & 0.10 \\
\hline $\mathrm{N}+$ & 34 & 0.19 & 0.03 & 0.17 & \\
\hline unknown & 1 & & & & \\
\hline \multicolumn{6}{|l|}{ Lymphovascular invasion } \\
\hline absent & 69 & 0.18 & 0.02 & 0.13 & 0.20 \\
\hline present & 23 & 0.19 & 0.04 & 0.16 & \\
\hline
\end{tabular}


Table 2. Cont.

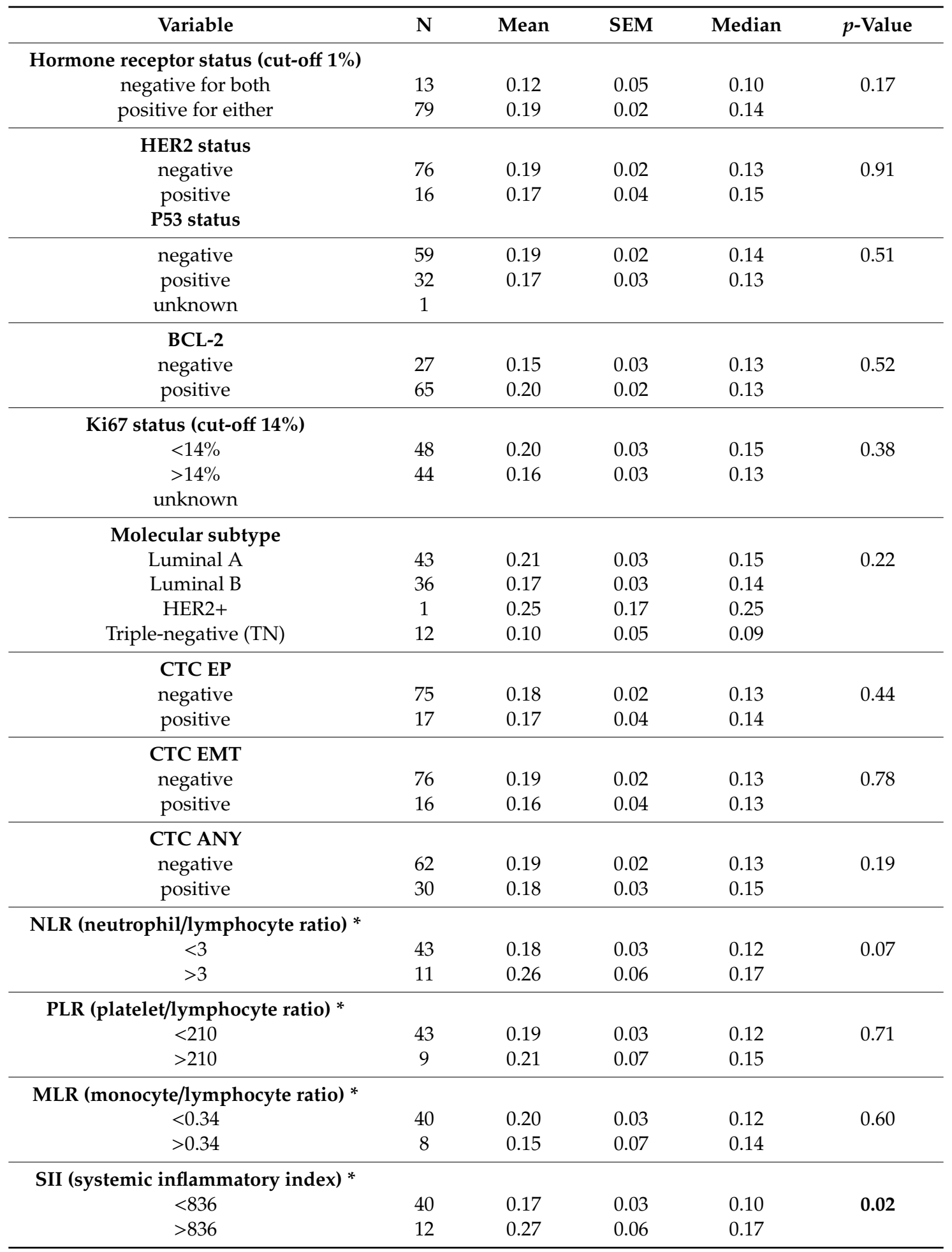

Abbreviations: CTC EP, circulating tumor cells with epithelial phenotype; CTC EMT, circulating tumor cells with epithelial-mesenchymal transition phenotype; CTC ANY, circulating tumor cells irrespective of phenotype. * Data for calculation of NLR, PLR, MLR, SII were available for 54, 52, 48, and 52 patients, respectively; NA, not applicable. $p$-Values $<0.05$ are written in Bold.

\subsection{Association between Nucleosomes and Plasma Cytokines}

Patients with nucleosomes above mean in peripheral blood had significantly elevated plasma IL-16 ( $p=0.005)$, IL-18 $(p=0.0004)$, and hepatocyte growth factor $(p=0.043)$, as compared to patients 
with nucleosomes below mean, while there was an inverse correlation between nucleosomes and IL-15 $(p=0.036)$. There was also a trend for higher IFN- $\alpha 2(p=0.055)$ and RANTES $(p=0.053)$ in patients with higher nucleosome level (Table 3).

Table 3. Association between nucleosomes and plasma cytokines.

\begin{tabular}{cccccc}
\hline Variable & $\mathbf{N}$ & Mean & SEM & Median & $p$-Value \\
\hline IFN_a2 (ng/mL) & & & & & \\
nucleosomes low & 57 & 101.6 & 3.2 & 102.2 & 0.055 \\
nucleosomes high & 26 & 114.5 & 4.7 & 114.7 & \\
\hline IL_16 (ng/mL) & & & & & \\
nucleosomes low & 58 & 349.2 & 19.8 & 330.9 & $\mathbf{0 . 0 0 5}$ \\
nucleosomes high & 27 & 446.7 & 29.0 & 419.5 & \\
\hline IL_18 (ng/mL) & & & & & \\
nucleosomes low & 57 & 60.4 & 12.9 & 33.8 & $\mathbf{0 . 0 0 0 4}$ \\
nucleosomes high & 26 & 120.0 & 19.1 & 69.7 & \\
\hline HGF (ng/mL) & & & & & \\
nucleosomes low & 58 & 760.8 & 184.7 & 222.7 & \\
nucleosomes high & 27 & 1312.2 & 270.8 & 438.6 & \\
\hline M_CSF (ng/mL) & & & & & \\
nucleosomes low & 58 & 10.7 & 1.5 & 6.9 & \\
nucleosomes high & 27 & 14.8 & 2.2 & 12.4 & \\
\hline IL_15 (ng/mL) & & & & & \\
nucleosomes low & 39 & 22.0 & 2.1 & 16.7 & 0.036 \\
nucleosomes high & 22 & 13.6 & 2.9 & 12.8 & \\
\hline RANTES (ng/mL) & & & & \\
nucleosomes low & 58 & 8890.2 & 816.2 & 7644.8 & 0.053 \\
nucleosomes high & 27 & 7185.2 & 1196.2 & 4352.3 & \\
\hline
\end{tabular}

Abbreviations: SEM, standard error of the mean. $p$-Values $<0.05$ are written in Bold.

\subsection{Nucleosomes and Coagulation}

There was no association between circulating nucleosomes and DD, TF, and/or uPA, while patients with nucleosomes above mean had significantly elevated levels of plasma PAI-1 (Table 4).

Table 4. Association between nucleosomes and coagulation.

\begin{tabular}{cccccc}
\hline Variable & $\mathbf{N}$ & Mean & SEM & Median & $p$-Value \\
\hline $\begin{array}{c}\text { Tissue factor }(\mathbf{p g} / \mathbf{m L}) \\
\text { nucleosomes low }\end{array}$ & 61 & 66.2 & 2.2 & 60.4 & 0.464 \\
nucleosomes high & 31 & 62.1 & 3.0 & 60.0 & \\
\hline D-dimer (ng/mL) & & & & & \\
nucleosomes low & 61 & 412.3 & 53.6 & 312.2 & 0.394 \\
nucleosomes high & 31 & 552.3 & 75.2 & 401.7 & \\
\hline uPA (ng/mL) & & & & & \\
nucleosomes low & 59 & 4.8 & 0.5 & 3.8 & \\
nucleosomes high & 31 & 4.6 & 0.6 & 3.7 & \\
\hline PAI_1 (pg/mL) & & & & & \\
nucleosomes low & 59 & 285.5 & 21.6 & 269.2 & $\mathbf{0 . 0 4 2}$ \\
nucleosomes high & 31 & 387.4 & 29.8 & 305.2 & \\
\hline
\end{tabular}

Abbreviations: SEM, standard error of the mean; uPA, urokinase plasminogen activator; PAI-1, plasminogen activator inhibitor-1. * uPA and PAI-1 were not determined in two patients. $p$-Values $<0.05$ are written in Bold. 


\subsection{Prognostic Value of Nucleosomes on Disease-Free Survival in Primary Breast Cancer}

At a median follow-up time of 55.0 months (range $=4.9-76.7$ months), 23 patients $(25.0 \%)$ had experienced a DFS event, and 15 patients $(16.3 \%)$ had died. Herein, we present DFS analysis due to the immaturity of overall survival data. Patients with lower than mean nucleosomes had significantly better disease-free survival ( $\mathrm{HR}=0.46,95 \% \mathrm{CI} 0.19-1.12, p=0.05$ ) (Figure 1 ). The prognostic value of circulating nucleosomes was most pronounced in lymph node-positive disease with high proliferation rate and in patients with detectable circulating tumor cells with epithelial-to-mesenchymal transition, but negative for epithelial circulating tumor cells (Table 5). In a multivariate analysis, nucleosomes, hormone receptor status, HER2 status, lymph node involvement, and tumor grade were independent predictors of disease-free survival (Table 6).

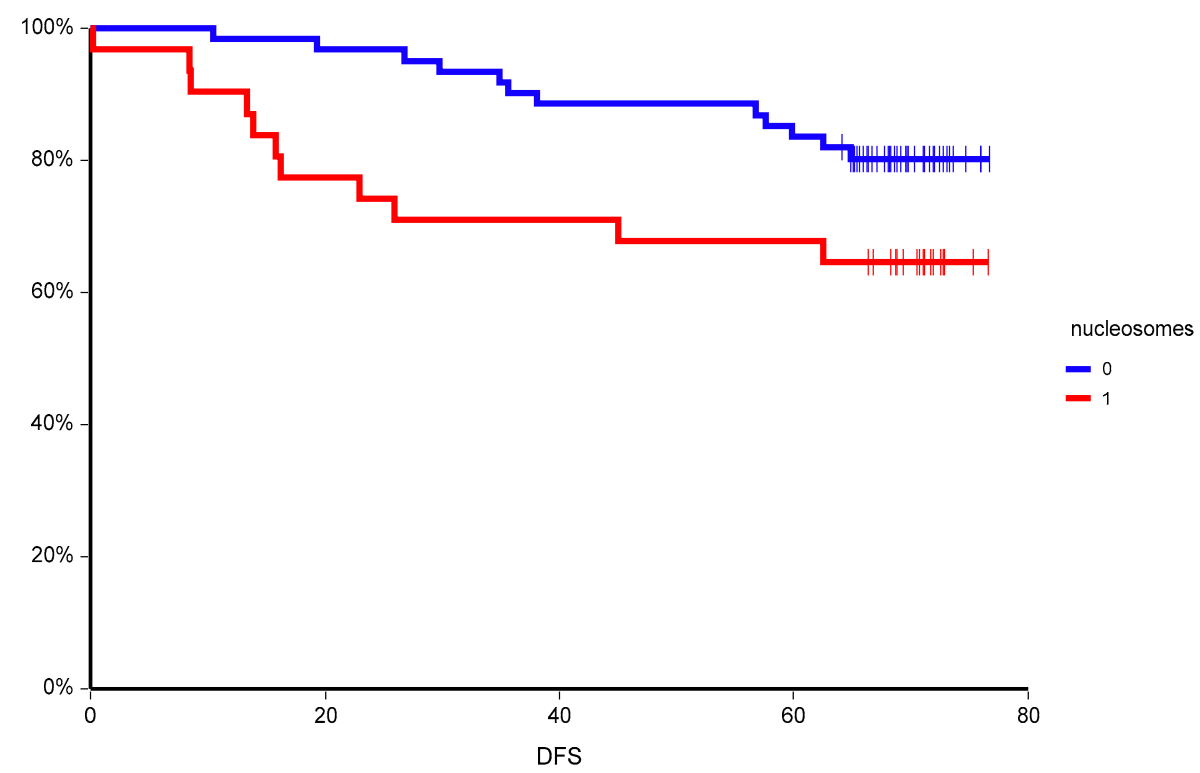

Figure 1. Kaplan-Meier estimates of probabilities of disease-free survival according to plasma nucleosome status in primary breast cancer patients $(n=92)$. HR $=0.46 .95 \%$ CI $0.19-1.12, p=0.05$, 0 -nucleosomes below mean, 1—nucleosomes above mean.

Table 5. Prognostic value of nucleosomes on disease-free survival in primary breast cancer (nucleosomes dichotomized below vs. above mean).

\begin{tabular}{ccccc}
\hline Variable & HR & 95\% CI Low & 95\% CI High & $p$-Value \\
\hline All & 0.46 & 0.19 & 1.12 & $\mathbf{0 . 0 5}$ \\
\hline T-stage & & & & \\
T1 & 0.29 & 0.08 & 1.02 & $\mathbf{0 . 0 4}$ \\
>T1 & 0.56 & 0.14 & 2.19 & 0.33 \\
\hline Histology & & & & \\
IDC & 0.35 & 0.14 & 0.91 & 0.01 \\
other & 0 & 0 & 0 & 0.09 \\
Grade & & & & 0.15 \\
\hline low & 0.31 & 0.07 & 1.34 & \\
high grade & 0.48 & 0.15 & 1.55 & 0.86 \\
Lymph nodes & & & & $\mathbf{0 . 0 3}$ \\
N0 & 0.86 & 0.15 & 4.92 & \\
N+ & 0.36 & 0.13 & 1.04 &
\end{tabular}


Table 5. Cont.

\begin{tabular}{|c|c|c|c|c|}
\hline Variable & HR & 95\% CI Low & 95\% CI High & $p$-Value \\
\hline \multicolumn{5}{|c|}{ Lymphovascular invasion } \\
\hline absent & 0.46 & 0.14 & 1.53 & 0.15 \\
\hline present & 0.54 & 0.15 & 1.97 & 0.31 \\
\hline \multicolumn{5}{|c|}{ Hormone receptor status (cut-off $1 \%$ ) } \\
\hline negative for both & 0.36 & 0.04 & 3.33 & 0.21 \\
\hline positive for either & 0.41 & 0.15 & 1.15 & 0.06 \\
\hline \multicolumn{5}{|l|}{ HER2 status } \\
\hline negative & 0.55 & 0.19 & 1.6 & 0.23 \\
\hline positive & 0.3 & 0.06 & 1.55 & 0.09 \\
\hline \multicolumn{5}{|l|}{ P53 status } \\
\hline negative & 0.48 & 0.16 & 1.44 & 0.13 \\
\hline positive & 0.39 & 0.08 & 1.86 & 0.20 \\
\hline \multicolumn{5}{|l|}{ BCL-2 } \\
\hline negative & 0.25 & 0.05 & 1.17 & 0.02 \\
\hline positive & 0.59 & 0.19 & 1.85 & 0.33 \\
\hline \multicolumn{5}{|c|}{ Ki67 status (cut-off $14 \%$ ) } \\
\hline$<14 \%$ & 0.73 & 0.11 & 4.71 & 0.72 \\
\hline$>14 \%$ & 0.35 & 0.12 & 1 & 0.02 \\
\hline \multicolumn{5}{|l|}{ CTC EP } \\
\hline negative & 0.31 & 0.12 & 0.83 & 0.01 \\
\hline positive & 0 & 0 & 0 & 0.18 \\
\hline \multicolumn{5}{|l|}{ СТС ЕМТ } \\
\hline negative & 0.68 & 0.23 & 2.03 & 0.46 \\
\hline positive & 0.17 & 0.03 & 0.9 & 0.01 \\
\hline \multicolumn{5}{|l|}{ CTC ANY } \\
\hline negative & 0.45 & 0.13 & 1.52 & 0.14 \\
\hline positive & 0.5 & 0.14 & 1.87 & 0.27 \\
\hline
\end{tabular}

$p$-Values $<0.05$ are written in Bold.

Table 6. Multivariate analysis of factors associated with disease-free survival.

\begin{tabular}{ccccc}
\hline Variable & HR & $\mathbf{9 5 \% ~ C I ~ L o w ~}$ & $\mathbf{9 5 \% ~ C I ~ H i g h ~}$ & $p$-Value \\
\hline $\begin{array}{c}\text { Nucleosomes } \\
\text { above mean vs. below mean }\end{array}$ & 2.67 & 1.12 & 6.36 & $\mathbf{0 . 0 2 6 8}$ \\
\hline $\begin{array}{c}\text { Hormone receptor status (cut-off 1\%) } \\
\text { positive for either vs. negative for both }\end{array}$ & 0.30 & 0.11 & 0.80 & $\mathbf{0 . 0 1 6 4}$ \\
\hline $\begin{array}{c}\text { HER2 status } \\
\text { amplified vs. nonamplified }\end{array}$ & 3.06 & 1.21 & 7.79 & $\mathbf{0 . 0 1 8 7}$ \\
\hline $\begin{array}{l}\text { Lymph nodes } \\
\text { positive vs. negative }\end{array}$ & 6.56 & 2.50 & 17.21 & $\mathbf{0 . 0 0 0 1}$ \\
\hline $\begin{array}{c}\text { Grade } \\
\text { grade 3 vs. grade 1 and 2 }\end{array}$ & 2.85 & 1.14 & 7.08 & $\mathbf{0 . 0 2 4 6}$ \\
\hline
\end{tabular}

$p$-Values $<0.05$ are written in Bold.

Circulating nucleosomes added prognostic value also to prognostic value of CTC_EMT, where double-positive patients (positive for both CTC_EMT and high-circulating nucleosomes) had worse prognosis compared to all other groups of patients (Figure 2). 


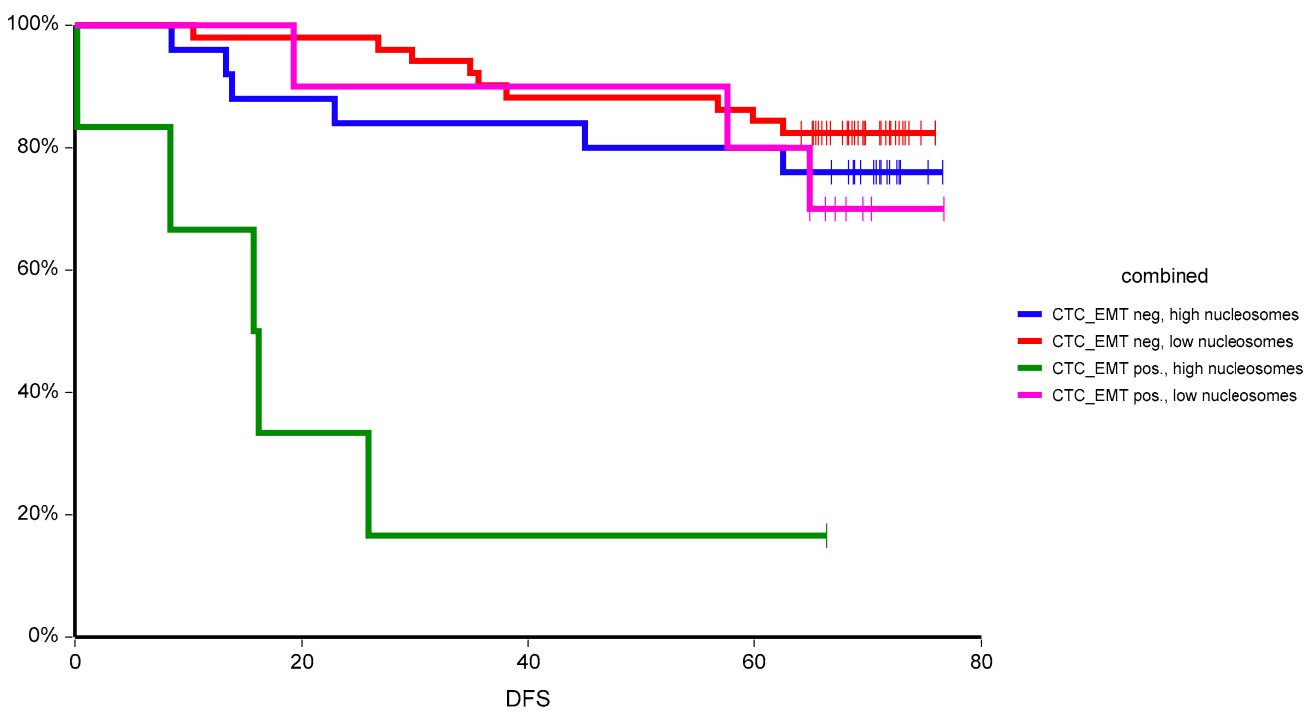

Figure 2. Kaplan-Meier estimates of probabilities of disease-free survival according to plasma nucleosome status and CTC_EMT in primary breast cancer patients $(n=92)$. Patients positive for CTC_EMT and high level of circulating nucleosomes had significantly worse survival compared to all other groups $(p=0.0000003)$.

\section{Discussion}

In this translational study, circulating nucleosomes showed neither an association with basic patient/tumor characteristics nor a correlation to CTCs. The origin of circulating nucleosomes is unclear and likely complex [25]. While there is no correlation between CTCs and SII and/or neutrophil/lymphocyte ratio $[23,24]$, this study showed for the first time an association between plasma nucleosomes and SII. Patients with high SII had significantly higher level of nucleosomes. Similarly, there was a trend of higher nucleosomes in patients with high neutrophil/lymphocyte ratio, however, the neutrophil/lymphocyte ratio is part of the SII.

Tumor-induced systemic changes in immune cells contribute to cancer progression and metastasis. Various forms of ecDNA including extracellular nucleosomes and naked ecDNA differ in their cytotoxic and proinflammatory effects [26]. For example, histones in the nucleosomes induce proinflammatory signaling via toll-like receptors (TLR2/4), with subsequent production of TNF- $\alpha$, IL-6, IL-10, and myeloperoxidase, but they exhibit TLR-independent cytotoxicity as well [26-28]. On the other hand, the ecDNA as part of the nucleosomes is recognized by the TLR9 [29]. In our study, nucleosomes were associated with several proinflammatory cytokines, suggesting the association of circulating nucleosomes with systemic inflammation. Histones in the nucleosomes could induce formation of neutrophil extracellular traps (NETs), which contain nucleosomes and stimulate further NETs production in a positive feedback loop [27]. On the other hand, nucleosomes could induce different inflammatory pathways, as they, in contrast to histones, seem not to be cytotoxic to the endothelium [28]. The analyzed nucleosomes could be from tumor cells, but also from the released NETs. This would explain the observed association between circulating nucleosomes and systemic inflammation in primary breast cancer patients. NETs contain nuclear DNA and proteins that possess antibacterial characteristics crucial for fighting pathogens [30,31]. The same NETs, however, also induce intravascular coagulation [32] and their overproduction can lead to autoimmune diseases [33]. While circulating ecDNA correlates with activation of coagulation [34], we for the first time describe this association for circulating nucleosomes. Further research is needed to uncover if nucleosomes directly activate PAI-1, or if high PAI-1 is a marker of coagulation activation in more aggressive disease that leads to release of more nucleosomes. 
Data on the prognostic value of plasma nucleosomes in breast cancer is limited. In a small study, nucleosomes were elevated in locally confined and metastatic breast cancer in comparison to healthy individuals. During neoadjuvant chemotherapy, patients with no change of a local disease had significantly higher pretherapeutic concentrations of nucleosomes than patients in remission [14]. In another study, plasma nucleosomes were higher in primary breast cancer patients when compared to healthy controls, and similarly to our study, there was no association between nucleosomes and patient/tumor characteristics [15]. Circulating nucleosomes were, however, not able to discriminate between benign and malignant breast lesions [35]. Their concentration was found to be associated with lymph node-positive breast cancer and the presence of distant metastases [35].

In our study, we observed an inferior outcome of primary breast cancer patients with high plasma nucleosomes. This is in contrast to a previous study, where elevated plasma nucleosomes were associated with a better prognosis in both node-negative and node-positive early breast cancer [15]. However, the nucleosome detection method as well as the cut-off value to discriminate "low" and "high" plasma nucleosomes was different compared to our trial and therefore, these differences in results could be due to these factors. In our trial, the prognostic value of nucleosomes was consistent in various subgroups, however, it was most pronounced in poor prognostic subgroups such as lymph node-positive disease with high proliferation rate and in patients with detectable circulating tumor cells with epithelial-to-mesenchymal transition. The prognostic value of circulating nucleosomes was independent from established prognostic markers and was confirmed in a multivariate analysis. Moreover, when we combined two circulating biomarkers, circulating tumor cells, and circulating nucleosomes, we were able to uncover a subgroup of patients with extremely poor prognosis with two-year DFS of only 33.3\%.

Our study has some limitations. The major one is small sample size, especially for associations between inflammatory indexes and nucleosomes. This is associated with decreased statistical power of analyses and increased confidence intervals of results. Other limitations represent the data availability for analysis of association between circulating nucleosomes and various clinic-pathological parameters, which further decreases statistical robustness and could have an impact on study results. Circulating plasma nucleosomes increase in non-neoplastic disease processes including inflammation, autoimmune diseases, sepsis, and stroke. When we analyzed association between chronic medication/comorbidities and circulating nucleosomes, no association was found, however, none of our patients received anti-inflammatory drugs and/or had inflammatory disease that could affect study results. Another limitation is lack of follow-up analysis on patient samples collected postsurgery to examine whether the presurgery baseline levels of circulating plasma nucleosomes were altered postsurgery and whether this alteration in circulating nucleosome levels is correlated with decrease in systemic inflammatory index.

\section{Conclusions}

In conclusion, in this translational study, we have shown for the first time that circulating nucleosomes are associated with systemic inflammation and activation of coagulation in primary breast cancer. More importantly, we proved their prognostic value. While it is clear that the underlying mechanisms of nucleosome release, their origin, and their fate require further studies, we suggest that the quantification of plasma nucleosomes could be added to the established prognostic markers in breast cancer. Future trials should focus on validation of these results to establish prognostic utility of plasma circulation nucleosomes in addition to established prognostic factors.

Author Contributions: Conceptualization, P.C., and M.M.; Data curation, G.M., T.S., and K.K.; Formal analysis, G.M., T.S., P.G., D.C., and P.C.; Funding acquisition, P.C., G.M., and M.M.; Investigation, M.K., G.M., J.B., and M.M.; Methodology, G.M., T.S., and P.C.; Project administration, P.C. and M.M.; Resources, J.M., K.K., M.K., J.B., and M.M.; Validation, P.C. and M.M.; Visualization, K.K., D.P., and J.M.; Writing-Original draft, P.C., and M.M.; Writing-Review \& editing, all authors. All authors have read and agreed to the published version of the manuscript. 
Funding: This research was funded by the Slovak Research and Development Agency (APVV), grant number APVV-16-0010, APVV-16-0178, by ERA-NET EuroNanoMed II INNOCENT and by Scientific Grant Agency (VEGA), contracts No. 1/0724/11, 1/0044/15, 1/0271/17, and 2/0052/18.

Acknowledgments: We would like to acknowledge Denisa Manasova for her excellent technical help. We are grateful to all patients for their participation in the study.

Conflicts of Interest: The authors declare no conflict of interest.

\section{Appendix A}

Table A1. Drug history in the last 6 months.

\begin{tabular}{ccc}
\hline Chronic Medication & $\mathbf{N}$ & $\mathbf{\%}$ \\
\hline NSAID & 0 & 0.0 \\
Corticosteroids & 0 & 0.0 \\
L-thyroxin & 8 & 8.7 \\
ACEi & 11 & 12.0 \\
Sartans & 14 & 15.2 \\
Betablockers & 27 & 29.3 \\
Statins & 15 & 16.3 \\
Metformin & 4 & 4.3 \\
Insulin & 2 & 2.2 \\
LMWH & 7 & 7.6 \\
Warfarin & 0 & 0.0 \\
\hline
\end{tabular}

Abbreviations: ACEi, angiotensin-converting enzyme inhibitors; LMWH, low-molecular-weight heparin.

\section{References}

1. Bray, F.; Ferlay, J.; Soerjomataram, I.; Siegel, R.L.; Torre, L.A.; Jemal, A. Global cancer statistics 2018: GLOBOCAN estimates of incidence and mortality worldwide for 36 cancers in 185 countries. CA Cancer J. Clin. 2018, 68, 394-424. [CrossRef]

2. McAnena, P.; Brown, J.A.; Kerin, M.J. Circulating Nucleosomes and Nucleosome Modifications as Biomarkers in Cancer. Cancers 2017, 9, 5. [CrossRef] [PubMed]

3. Lui, Y.Y.; Chik, K.W.; Chiu, R.W.; Ho, C.Y.; Lam, C.W.; Lo, Y.M. Predominant hematopoietic origin of cell-free DNA in plasma and serum after sex-mismatched bone marrow transplantation. Clin. Chem. 2002, 48, 421-427. [CrossRef] [PubMed]

4. Sun, K.; Jiang, P.; Chan, K.C.; Wong, J.; Cheng, Y.K.; Liang, R.H.; Chan, W.K.; Ma, E.S.; Chan, S.L.; Cheng, S.H.; et al. Plasma DNA tissue mapping by genome-wide methylation sequencing for noninvasive prenatal, cancer, and transplantation assessments. Proc. Natl. Acad. Sci. USA 2015, 112, E5503-E5512. [CrossRef] [PubMed]

5. Kustanovich, A.; Schwartz, R.; Peretz, T.; Grinshpun, A. Life and death of circulating cell-free DNA. Cancer Biol. 2019, 20, 1057-1067. [CrossRef] [PubMed]

6. Khorasanizadeh, S. The nucleosome: From genomic organization to genomic regulation. Cell 2004, 116, 259-272. [CrossRef]

7. Jiang, N.; Reich, C.F., 3rd; Monestier, M.; Pisetsky, D.S. The expression of plasma nucleosomes in mice undergoing in vivo apoptosis. Clin. Immunol. 2003, 106, 139-147. [CrossRef]

8. Holdenrieder, S.; Nagel, D.; Schalhorn, A.; Heinemann, V.; Wilkowski, R.; von Pawel, J.; Raith, H.; Feldmann, K.; Kremer, A.E.; Muller, S.; et al. Clinical relevance of circulating nucleosomes in cancer. Ann. N.Y. Acad. Sci. 2008, 1137, 180-189. [CrossRef]

9. Zeerleder, S.; Zwart, B.; Wuillemin, W.A.; Aarden, L.A.; Groeneveld, A.B.; Caliezi, C.; van Nieuwenhuijze, A.E.; van Mierlo, G.J.; Eerenberg, A.J.; Lammle, B.; et al. Elevated nucleosome levels in systemic inflammation and sepsis. Crit. Care Med. 2003, 31, 1947-1951. [CrossRef]

10. Pisetsky, D.S. The complex role of DNA, histones and HMGB1 in the pathogenesis of SLE. Autoimmunity 2014, 47, 487-493. [CrossRef] 
11. Bauden, M.; Pamart, D.; Ansari, D.; Herzog, M.; Eccleston, M.; Micallef, J.; Andersson, B.; Andersson, R. Circulating nucleosomes as epigenetic biomarkers in pancreatic cancer. Clin. Epigenet. 2015, 7, 106. [CrossRef] [PubMed]

12. Rahier, J.F.; Druez, A.; Faugeras, L.; Martinet, J.P.; Gehenot, M.; Josseaux, E.; Herzog, M.; Micallef, J.; George, F.; Delos, M.; et al. Circulating nucleosomes as new blood-based biomarkers for detection of colorectal cancer. Clin. Epigenet. 2017, 9, 53. [CrossRef] [PubMed]

13. Wang, K.; Shan, S.; Wang, S.; Gu, X.; Zhou, X.; Ren, T. HMGB1-containing nucleosome mediates chemotherapy-induced metastasis of human lung cancer. Biochem. Biophys. Res. Commun. 2018, 500, 758-764. [CrossRef] [PubMed]

14. Stoetzer, O.J.; Fersching, D.M.; Salat, C.; Steinkohl, O.; Gabka, C.J.; Hamann, U.; Braun, M.; Feller, A.M.; Heinemann, V.; Siegele, B.; et al. Prediction of response to neoadjuvant chemotherapy in breast cancer patients by circulating apoptotic biomarkers nucleosomes, DNAse, cytokeratin-18 fragments and survivin. Cancer Lett. 2013, 336, 140-148. [CrossRef] [PubMed]

15. Kuroi, K.; Tanaka, C.; Toi, M. Plasma Nucleosome Levels in Node-Negative Breast Cancer Patients. Breast Cancer 1999, 6, 361-364. [CrossRef] [PubMed]

16. Wittwer, C.; Boeck, S.; Heinemann, V.; Haas, M.; Stieber, P.; Nagel, D.; Holdenrieder, S. Circulating nucleosomes and immunogenic cell death markers HMGB1, sRAGE and DNAse in patients with advanced pancreatic cancer undergoing chemotherapy. Int. J. Cancer 2013, 133, 2619-2630. [CrossRef] [PubMed]

17. Holdenrieder, S.; Stieber, P.; von Pawel, J.; Raith, H.; Nagel, D.; Feldmann, K.; Seidel, D. Circulating nucleosomes predict the response to chemotherapy in patients with advanced non-small cell lung cancer. Clin. Cancer Res. 2004, 10, 5981-5987. [CrossRef] [PubMed]

18. Mego, M.; Karaba, M.; Minarik, G.; Benca, J.; Silvia, J.; Sedlackova, T.; Manasova, D.; Kalavska, K.; Pindak, D.; Cristofanilli, M.; et al. Circulating Tumor Cells With Epithelial-to-mesenchymal Transition Phenotypes Associated With Inferior Outcomes in Primary Breast Cancer. Anticancer Res. 2019, 39, 1829-1837. [CrossRef]

19. Mego, M.; Cierna, Z.; Janega, P.; Karaba, M.; Minarik, G.; Benca, J.; Sedlackova, T.; Sieberova, G.; Gronesova, P.; Manasova, D.; et al. Relationship between circulating tumor cells and epithelial to mesenchymal transition in early breast cancer. BMC Cancer 2015, 15, 533. [CrossRef]

20. Mego, M.; Mani, S.A.; Lee, B.N.; Li, C.; Evans, K.W.; Cohen, E.N.; Gao, H.; Jackson, S.A.; Giordano, A.; Hortobagyi, G.N.; et al. Expression of epithelial-mesenchymal transition-inducing transcription factors in primary breast cancer: The effect of neoadjuvant therapy. Int. J. Cancer 2012, 130, 808-816. [CrossRef]

21. Mego, M.; Karaba, M.; Minarik, G.; Benca, J.; Sedlackova, T.; Tothova, L.; Vlkova, B.; Cierna, Z.; Janega, P.; Luha, J.; et al. Relationship between circulating tumor cells, blood coagulation, and urokinase-plasminogen-activator system in early breast cancer patients. Breast J. 2015, 21, 155-160. [CrossRef]

22. Mego, M.; Cholujova, D.; Minarik, G.; Sedlackova, T.; Gronesova, P.; Karaba, M.; Benca, J.; Cingelova, S.; Cierna, Z.; Manasova, D.; et al. CXCR4-SDF-1 interaction potentially mediates trafficking of circulating tumor cells in primary breast cancer. BMC Cancer 2016, 16, 127. [CrossRef] [PubMed]

23. De Giorgi, U.; Mego, M.; Scarpi, E.; Giordano, A.; Giuliano, M.; Valero, V.; Alvarez, R.H.; Ueno, N.T.; Cristofanilli, M.; Reuben, J.M. Association between circulating tumor cells and peripheral blood monocytes in metastatic breast cancer. Adv. Med. Oncol. 2019, 11, 1758835919866065. [CrossRef] [PubMed]

24. Miklikova, S.; Minarik, G.; Sedlackova, T.; Plava, J.; Cihova, M.; Jurisova, S.; Kalavska, K.; Karaba, M.; Benca, J.; Smolkova, B.; et al. Inflammation-Based Scores Increase the Prognostic Value of Circulating Tumor Cells in Primary Breast Cancer. Cancers 2020, 12, 1134. [CrossRef] [PubMed]

25. Thierry, A.R.; El Messaoudi, S.; Gahan, P.B.; Anker, P.; Stroun, M. Origins, structures, and functions of circulating DNA in oncology. Cancer Metastasis Rev. 2016, 35, 347-376. [CrossRef] [PubMed]

26. Chaudhary, S.; Mittra, I. Cell-free chromatin: A newly described mediator of systemic inflammation. J. Biosci. 2019, 44, 32. [CrossRef] [PubMed]

27. Abrams, S.T.; Zhang, N.; Manson, J.; Liu, T.; Dart, C.; Baluwa, F.; Wang, S.S.; Brohi, K.; Kipar, A.; Yu, W.; et al. Circulating histones are mediators of trauma-associated lung injury. Am. J. Respir. Crit. Care Med. 2013, 187, 160-169. [CrossRef]

28. Xu, J.; Zhang, X.; Pelayo, R.; Monestier, M.; Ammollo, C.T.; Semeraro, F.; Taylor, F.B.; Esmon, N.L.; Lupu, F.; Esmon, C.T. Extracellular histones are major mediators of death in sepsis. Nat. Med. 2009, 15, 1318-1321. [CrossRef] 
29. Kang, T.H.; Mao, C.P.; Kim, Y.S.; Kim, T.W.; Yang, A.; Lam, B.; Tseng, S.H.; Farmer, E.; Park, Y.M.; Hung, C.F. TLR9 acts as a sensor for tumor-released DNA to modulate anti-tumor immunity after chemotherapy. J. Immunother. Cancer 2019, 7, 260. [CrossRef]

30. Yang, H.; Biermann, M.H.; Brauner, J.M.; Liu, Y.; Zhao, Y.; Herrmann, M. New Insights into Neutrophil Extracellular Traps: Mechanisms of Formation and Role in Inflammation. Front. Immunol. 2016, 7, 302. [CrossRef]

31. Brinkmann, V.; Reichard, U.; Goosmann, C.; Fauler, B.; Uhlemann, Y.; Weiss, D.S.; Weinrauch, Y.; Zychlinsky, A. Neutrophil extracellular traps kill bacteria. Science 2004, 303, 1532-1535. [CrossRef] [PubMed]

32. McDonald, B.; Davis, R.P.; Kim, S.J.; Tse, M.; Esmon, C.T.; Kolaczkowska, E.; Jenne, C.N. Platelets and neutrophil extracellular traps collaborate to promote intravascular coagulation during sepsis in mice. Blood 2017, 129, 1357-1367. [CrossRef] [PubMed]

33. Kaplan, M.J.; Radic, M. Neutrophil extracellular traps: Double-edged swords of innate immunity. J. Immunol. 2012, 189, 2689-2695. [CrossRef]

34. Von Meijenfeldt, F.A.; Burlage, L.C.; Bos, S.; Adelmeijer, J.; Porte, R.J.; Lisman, T. Elevated Plasma Levels of Cell-Free DNA During Liver Transplantation Are Associated With Activation of Coagulation. Liver Transpl. 2018, 24, 1716-1725. [CrossRef] [PubMed]

35. Roth, C.; Pantel, K.; Muller, V.; Rack, B.; Kasimir-Bauer, S.; Janni, W.; Schwarzenbach, H. Apoptosis-related deregulation of proteolytic activities and high serum levels of circulating nucleosomes and DNA in blood correlate with breast cancer progression. BMC Cancer 2011, 11, 4. [CrossRef] [PubMed]

(C) 2020 by the authors. Licensee MDPI, Basel, Switzerland. This article is an open access article distributed under the terms and conditions of the Creative Commons Attribution (CC BY) license (http://creativecommons.org/licenses/by/4.0/). 